IJMMS 26:2 (2001) 117-121

PII. S0161171201005397

http://ijmms.hindawi.com

(c) Hindawi Publishing Corp.

\title{
NEAR FRATTINI SUBGROUPS OF RESIDUALLY FINITE GENERALIZED FREE PRODUCTS OF GROUPS
}

\author{
MOHAMMAD K. AZARIAN
}

(Received 3 May 1999 and in revised form 9 June 2000)

\begin{abstract}
Let $G=A \star_{H} B$ be the generalized free product of the groups $A$ and $B$ with the amalgamated subgroup $H$. Also, let $\lambda(G)$ and $\psi(G)$ represent the lower near Frattini subgroup and the near Frattini subgroup of $G$, respectively. If $G$ is finitely generated and residually finite, then we show that $\psi(G) \leq H$, provided $H$ satisfies a nontrivial identical relation. Also, we prove that if $G$ is residually finite, then $\lambda(G) \leq H$, provided: (i) $H$ satisfies a nontrivial identical relation and $A, B$ possess proper subgroups $A_{1}, B_{1}$ of finite index containing $H$; (ii) neither $A$ nor $B$ lies in the variety generated by $H$; (iii) $H<A_{1} \leq A$ and $H<B_{1} \leq B$, where $A_{1}$ and $B_{1}$ each satisfies a nontrivial identical relation; (iv) $H$ is nilpotent.

2000 Mathematics Subject Classification. 20E06, 20E28.
\end{abstract}

1. Definitions and notation. Throughout the paper our notation will be standard. We use $G=A \star_{H} B$ to represent the generalized free product of the groups $A$ and $B$ with the amalgamated subgroup $H$, as in B. H. Neumann [12]. A group $G$ is residually finite if every nontrivial element of $G$ can be excluded from some normal subgroup of finite index in $G$. An $N$-group is a finite group in which the normalizer of every nontrivial solvable subgroup is solvable. A group $G$ is called 3-metabelian if every subgroup of $G$ which is generated by 3 elements is metabelian. A variety of groups is the collection of all groups satisfying a given set of identical relations or laws. The core of the subgroup $H$ in $G$ is represented by $K(G, H)$.

An element $g$ of a group $G$ is a near generator of $G$ if there exists a subset $S$ of $G$ such that $|G:\langle S\rangle|$ is infinite, but $|G:\langle g, S\rangle|$ is finite. Thus, an element $g$ of $G$ is a non-near generator of $G$ if for every subset $S$ of $G$, finiteness of $|G:\langle g, S\rangle|$ implies finiteness of $|G:\langle S\rangle|$. A subgroup $M$ of a group $G$ is nearly maximal in $G$ if $|G: M|$ is infinite, but $|G: N|$ is finite, whenever $M<N \leq G$. That is, $M$ is nearly maximal in $G$ if it is maximal with respect to being of infinite index in $G$. The set of all non-near generators of a group $G$ forms a characteristic subgroup called the lower near Frattini subgroup of $G$, denoted by $\lambda(G)$. The intersection of all nearly maximal subgroups of $G$ is called the upper near Frattini subgroup of $G$, denoted by $\mu(G)$. If there are no nearly maximal subgroups, then $\mu(G)=G$. In general, $\lambda(G) \leq \mu(G)$. If $\lambda(G)=\mu(G)$, then their common value is called the near Frattini subgroup of $G$, denoted by $\psi(G)$. Definitions concerning the near Frattini subgroup are due to J. B. Riles [13].

2. Background and history. In response to a question raised by N. Itô concerning the existence of maximal subgroups in free products of groups, G. Higman and B. H. Neumann proved that the Frattini subgroup of a free product of (nontrivial) groups is 
the trivial group [11, Theorem 2, page 87]. That is, they showed that free products of groups do have maximal subgroups. They extended Itô's question and asked whether a generalized free product of groups necessarily has maximal subgroups. They asked whether or not the Frattini subgroup of a generalized free product of groups is contained in the amalgamated subgroup. These questions have been answered for some certain classes of generalized free products of groups (see [2, 3, 4, 5, 6, 7, 8, 9]).

Similar results for the (lower) near Frattini subgroups of such generalized free products of groups are produced in [2, 3, 4, 5, 6, 7, 8, 9]. In this paper which is motivated by R. B. J. T. Allenby and C. Y. Tang [1], we continue our investigation to produce more results concerning the relationship between the (lower) near Frattini subgroup and the amalgamated subgroup of these generalized free products. In particular, in Section 3 we show that if $G=A \star_{H} B$ is finitely generated and residually finite, then $\psi(G) \leq H$, provided $H$ satisfies a nontrivial identical relation. Also, when $G=A \star_{H} B$ is residually finite, we prove that $\lambda(G) \leq H$, if any of the following conditions is satisfied: (i) $H$ satisfies a nontrivial identical relation and $A, B$ possess proper subgroups $A_{1}, B_{1}$ of finite index containing $H$; (ii) neither $A$ nor $B$ lies in the variety generated by $H$; (iii) $H<A_{1} \leq A$ and $H<B_{1} \leq B$, where $A_{1}$ and $B_{1}$ each satisfies a nontrivial identical relation; (iv) $H$ is nilpotent.

3. Results. Before tackling the new results, we need to state some known results from previous works.

THEOREM 3.1 [5, Theorem 3.6, page 502]. Let $G=A \star_{H} B$. If $H$ satisfies the minimum condition on subgroups, then $\lambda(G) \leq K(G, H)$.

THEOREM 3.2 [7, Theorem 4.2, page 6]. Let $G=A \star_{H} B$. If there exists a nontrivial normal subgroup $N$ of $G$ such that $N \cap H=1$, then $\lambda(G) \leq H$.

Proposition 3.3 [7, Proposition 4.6, page 6]. Let $G=A \star_{H} B$. If $\lambda(G) \cap A=\lambda(G) \cap B$ $=\lambda(G) \cap H$, then $\lambda(G) \leq H$.

Theorem 3.4 [7, Theorem 4.7, page 6]. Let $G=A \star_{H} B$. Suppose $A_{1}$ and $B_{1}$ are finite normal subgroups of $A$ and $B$, respectively. If $A_{1} \cap H=B_{1} \cap H$, and at least one of $A_{1}$ or $B_{1}$ is not contained in $H$, then $\lambda(G) \leq H$.

THEOREM 3.5 [9, Theorem 3.12, page 608]. Let $G=A \star_{H} B$ be residually finite. If $|A: H|=|B: H|=2$, then $\lambda(G) \leq K(G, H)$.

THEOREM 3.6 [7, Theorem 4.11, page 7]. Let $G=A \star_{H} B$. If $A$ and $B$ are countable groups, then $\lambda(G) \leq H$.

THEOREM 3.7. Let $G=A \star_{H} B$ be finitely generated and residually finite. If $H$ satisfies a nontrivial identical relation, then $\psi(G) \leq H$.

REMARK 3.8. We could refer to Theorem 3.6 and accept Theorem 3.7 without a proof. However, we present a direct proof, independent of the proof of Theorem 3.6.

Proof. Since $G$ is finitely generated by J. B. Riles [13, Proposition 1, page 157], $\lambda(G)=\mu(G)=\psi(G)$. Therefore, it is enough to show that $\lambda(G) \leq H$. If $\lambda(G) \cap A=$ 
$\lambda(G) \cap B=\lambda(G) \cap H$, then Proposition 3.3 is applicable. Otherwise, at least one of $\lambda(G) \cap A$ or $\lambda(G) \cap B$ properly contains $\lambda(G) \cap H$. If $|A: H|=|B: H|=2$, then by Theorem 3.5, $\lambda(G) \leq H$. Therefore, without loss of generality, we may assume that $|A: H|>2$. Thus, there must exist an element $a \in A$ such that $a \in \lambda(G)$ but $a \notin H$. Also, we let $b \in B \backslash H$ and $a_{1} \in A \backslash H \cup a H$. Now, since $a_{1}^{-1} a \notin H$, we conclude that $u=a_{1}^{-1}\left(a b^{-1} a b\right) a_{1} \in \lambda(G)$, where in reduced form the initial and final letters of $u$ are in $A \backslash H$.

Since $\lambda(G)$ is characteristic in $G$, the rest of the proof is very similar to the proof of Theorem 2 of R. B. J. T. Allenby and C. Y. Tang [1, page 302]. Thus, we use the same notation and set up as in [1] and we replace $\Phi(G)$ by $\lambda(G)$. In particular, we let $S=\left\langle u, b^{-1} u b\right\rangle, w\left(x_{1}, x_{2}, \ldots, x_{n}\right), w\left(y_{1}, y_{2}, \ldots, y_{n}\right), N, U, V, \bar{A}, \bar{B}, \bar{H}, \bar{G}$, and the natural map $\psi$, be as in the proof of Theorem 2 of [1]. To complete the proof we use the fact that $G$ is residually finite, Theorem 3.4, as well as the fact that the natural homomorphism takes a non-near generator of $G$ to a non-near generator of $\bar{G}$.

Theorem 3.7 can be applied to various residually finite generalized free products of groups. For example, if $G=A \star_{H} B$ is residually finite and is finitely generated, then $\psi(G) \leq H$, provided: (i) $H$ is of finite exponent, $H$ is periodic or $H$ is an $N$-group; (ii) $H$ is the ordinary free product of two cyclic groups of order 2; (iii) $H$ is metabelian, or $H$ is 3-metabelian; (iv) $H$ is nilpotent.

THEOREM 3.9. Let $G=A \star_{H} B$ be residually finite. If $H$ satisfies a nontrivial identical relation and if $A, B$ possess proper subgroups $A_{1}, B_{1}$ of finite index containing $H$, then $\lambda(G) \leq H$.

Proof. The first part of the proof is similar to the proof of Theorem 3 of R. B. J. T. Allenby and C. Y. Tang [1, page 302], and we note that $A_{1}, B_{1}$ here correspond to $K, L$ in [1]. Thus, if $U=N \cap A \cap \lambda(G) \triangleleft A$ and $V=N \cap B \cap \lambda(G) \triangleleft B$, are as in [1], where $\Phi(G)$ is replaced by $\lambda(G)$, then it is enough to show that $H U \neq A$ and $H V \neq B$. If this is not the case, then without loss of generality, we may assume that $H U=A$. Now, from the fact that $H \leq A_{1}<A$ and $\left|A: A_{1}\right|<\infty$, we deduce that $A_{1}(\lambda(G) \cap A) \geq H U=A$. This implies that

$$
A=\left\langle A_{1}, \lambda_{1}, \lambda_{2}, \lambda_{3}, \ldots, \lambda_{n}\right\rangle,
$$

where $\lambda_{1}, \lambda_{2}, \lambda_{3}, \ldots, \lambda_{n}$ are nontrivial and distinct elements of $\lambda(G)$. Thus,

$$
G=\langle A, B\rangle=\left\langle\lambda_{1}, \lambda_{2}, \lambda_{3}, \ldots, \lambda_{n}, A_{1}, B\right\rangle .
$$

Hence,

$$
\left|G:\left\langle\lambda_{1}, \lambda_{2}, \lambda_{3}, \ldots, \lambda_{n}, A_{1}, B\right\rangle\right|<\infty .
$$

But, since $\lambda_{1}, \lambda_{2}, \lambda_{3}, \ldots, \lambda_{n}$ are non-near generators of $G$, we must have $\left|G:\left\langle A_{1}, B\right\rangle\right|<\infty$. However, $\left|G:\left\langle A_{1}, B\right\rangle\right|<\infty$, is not possible. For if we take $a \in A \backslash A_{1}$ and $b \in B \backslash H$, then

$$
a b\left\langle A_{1}, B\right\rangle,(a b)^{2}\left\langle A_{1}, B\right\rangle, \ldots,(a b)^{n}\left\langle A_{1}, B\right\rangle, \ldots
$$

are incongruent $\bmod \left\langle A_{1}, B\right\rangle$. That is, $\left\langle A_{1}, B\right\rangle$ has infinitely many distinct cosets in $G$. Therefore, the assumption that $H U=A$ is reached to a contradiction, and thus, the proof is complete. 
THEOREM 3.10. Let $G=A \star_{H} B$ be residually finite. If neither $A$ nor $B$ lies in the variety generated by $H$, then $\lambda(G) \leq H$.

Proof. First we note that $H$ must satisfy a nontrivial identical relation. Otherwise, $H$ generates the variety of all groups, and thus it must contain both $A$ and $B$, contradicting the statement of the theorem. Also, since $G$ is residually finite, it contains a collection of normal subgroups $N_{\lambda}(\lambda \in \Lambda)$ of finite index such that $\bigcap_{\lambda \in \Lambda} N_{\lambda}=1$. If there exist $\mu, \nu \in \Lambda$ such that $H\left(A \cap N_{\mu}\right) \neq A$ and $H\left(B \cap N_{\nu}\right) \neq B$, then by Theorem 3.9, $\lambda(G) \leq H$. On the other hand, if $H\left(A \cap N_{\lambda}\right)=A$ for all $\lambda \in \Lambda$ and $H\left(B \cap N_{\lambda}\right)=B$, for all $\lambda \in \Lambda$, then again, by the argument given by R. B. J. T. Allenby and C. Y. Tang in the proof of the Frattini version of this theorem [1, Theorem 3, page 303], we conclude that $\lambda(G) \leq H$. If $\lambda(G) \npreceq H$, then we must have either $H\left(A \cap N_{\lambda}\right)=A$ for all $\lambda \in \Lambda$ or $H\left(B \cap N_{\lambda}\right)=B$, for all $\lambda \in A$, but not both. Hence, either $A$ or $B$ must satisfy the same identical relation as the amalgamated subgroup $H$, which is impossible, by the statement of the theorem. Therefore, we must have $\lambda(G) \leq H$, as desired.

THEOREM 3.11. Let $G=A \star_{H} B$ be residually finite. If $H<A_{1} \leq A$ and $H<B_{1} \leq B$, where $A_{1}$ and $B_{1}$ each satisfies a nontrivial identical relation, then $\lambda(G) \leq H$.

Proof. Since $H$ satisfies a nontrivial identical relation, if $G$ is finitely generated, then Theorem 3.6 is applicable. Also, if both $A_{1}$ and $B_{1}$ are of finite indices in $A$ and $B$, respectively, then again by Theorem 3.9, $\lambda(G) \leq H$. Now, since both $\lambda(G)$ and $\Phi(G)$ are characteristic subgroups of $G$, the proof of the general case is very similar to the proof of the Frattini version of this theorem by R. B. J. T. Allenby and C. Y. Tang [1, Theorem 1, page 303], and is left to the reader.

THEOREM 3.12. Let $G=A \star_{H} B$ be residually finite. If $H$ is nilpotent, then $\lambda(G) \leq H$.

Proof. If $G$ is finitely generated, then Theorem 3.7 is applicable. Otherwise, we use the same setup and notation as in the proof of the Frattini version of this theorem [1, Theorem 5, page 303] by R. B. J. T. Allenby and C. Y. Tang. To complete the proof, we use the fact that the natural homomorphism $\psi$ takes a non-near generator of $G$ to a non-near generator of its factor group $\bar{G}$, and we apply Theorem 3.1 as well.

As an immediate consequence of Theorem 3.12 and Theorem 7 of G. Baumslag [10, page 196], we have the following corollary.

COROLLARY 3.13. Let $G=A \star_{H} B$. If $A$ and $B$ are free groups and $H$ is cyclic, then $\lambda(G) \leq H$.

From our study of residually finite generalized free products of groups and their lower near Frattini subgroups in this paper, as well as $[8,9]$, we suspect that if the amalgamated subgroup satisfies a nontrivial identical relation, then the lower near Frattini subgroup of such generalized free products is contained in the amalgamated subgroup. Therefore, we make the following conjecture.

CONJECTURE 3.14. Let $G=A \star_{H} B$ be residually finite. If $H$ satisfies a nontrivial identical relation, then $\lambda(G) \leq H$. 
ACKNOWLEDGement. This work was supported in part by a grant from the University of Evansville Faculty Fellowship.

\section{REFERENCES}

[1] R. B. J. T. Allenby and C. Y. Tang, On the Frattini subgroup of a residually finite generalized free product, Proc. Amer. Math. Soc. 47 (1975), 300-304. MR 52\#10892. Zbl 322.20018.

[2] M. K. Azarian, On the near Frattini subgroups of amalgamated free products of groups, Houston J. Math. 16 (1990), no. 4, 523-528. MR 92d:20032. Zbl 742.20030.

[3] _ On the lower near Frattini subgroups of generalized free products with cyclic amalgamations, Houston J. Math. 17 (1991), no. 3, 419-423. MR 92j:20018a. Zbl 743.20020.

[4]___ On the near Frattini subgroup of the amalgamated free product of finitely generated abelian groups, Houston J. Math. 17 (1991), no. 3, 425-427. MR 92j:20018b. Zbl 743.20021.

[5]___ On the lower near Frattini subgroups of amalgamated free products of groups, Houston J. Math. 19 (1993), no. 4, 499-504. MR 94k:20046. Zbl 799.20028.

[6] _ On the near Frattini subgroups of certain groups, Houston J. Math. 20 (1994), no. 3, 555-560. MR 95d:20046. Zbl 817.20028.

[7] _ A key theorem on the near Frattini subgroups of generalized free product of groups, Houston J. Math. 22 (1996), no. 1, 1-10. MR 98b:20036. Zbl 855.20026.

[8]___ On the near Frattini subgroup of the generalized free product of finitely generated nilpotent groups, Houston J. Math. 23 (1997), no. 4, 613-615. MR 2000e:20052a. Zbl 896.20019.

[9] _ - On the near Frattini subgroups of amalgamated free products with residual properties, Houston J. Math. 23 (1997), no. 4, 603-612. MR 2000e:20052b. Zbl 896.20020.

[10] G. Baumslag, On the residual finiteness of generalized free products of nilpotent groups, Trans. Amer. Math. Soc. 106 (1963), 193-209. MR 26\#2489. Zbl 112.25904.

[11] G. Higman and B. H. Neumann, On two questions of Itô, J. London Math. Soc. 29 (1954), 84-88. MR 15,286g. Zbl 055.01602.

[12] B. H. Neumann, An essay on free products of groups with amalgamations, Philos. Trans. Roy. Soc. London Ser. A. 246 (1954), 503-554. MR 16,10d. Zbl 057.01702.

[13] J. B. Riles, The near Frattini subgroups of infinite groups, J. Algebra 12 (1969), 155-171. MR 39\#322. Zbl 182.03702.

MOHAMmad K. AZARIAN: DePartment of MATHEMATICS, UNiVERSity OF EVANSVILle, 1800 LINCOLN AVENUE, EVANSVILLE, IN 47722, USA

E-mail address: azarian@evansvi11e.edu 


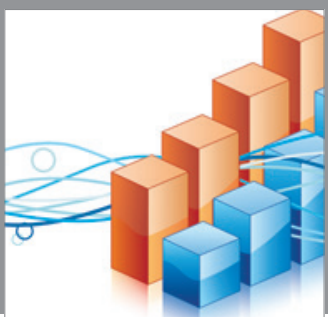

Advances in

Operations Research

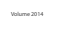

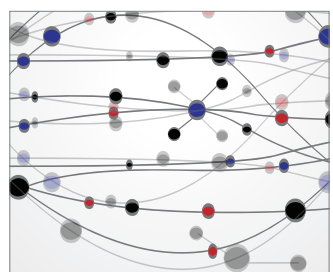

\section{The Scientific} World Journal
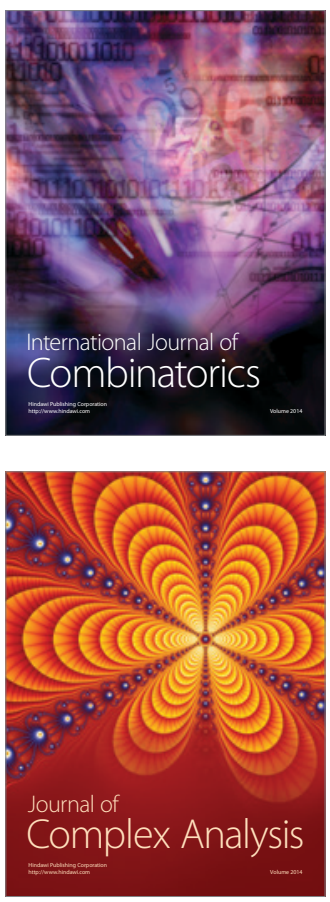

International Journal of

Mathematics and

Mathematical

Sciences
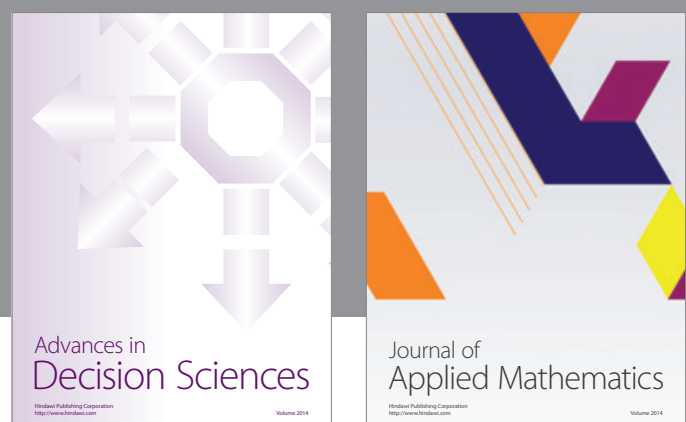

Journal of

Applied Mathematics
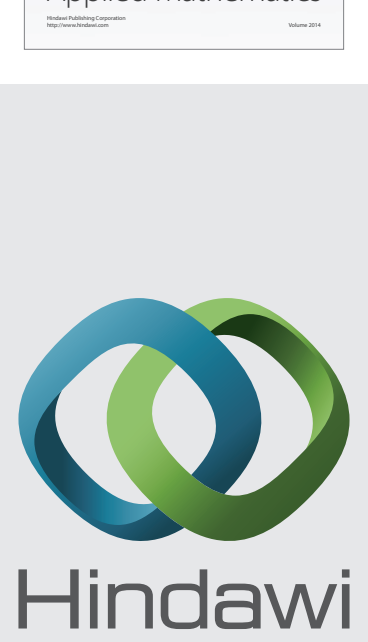

Submit your manuscripts at http://www.hindawi.com
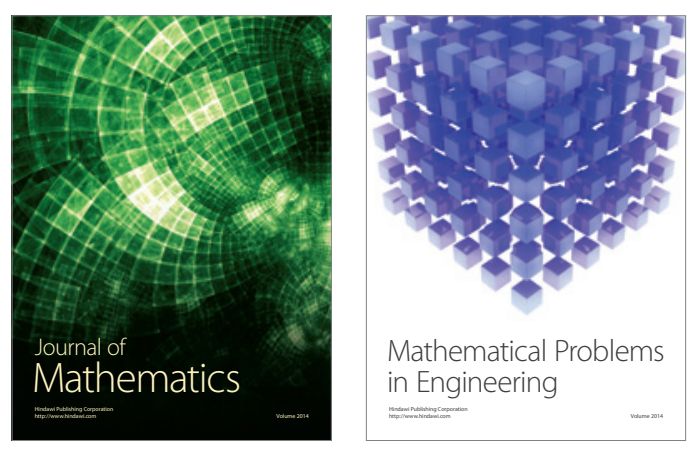

Mathematical Problems in Engineering
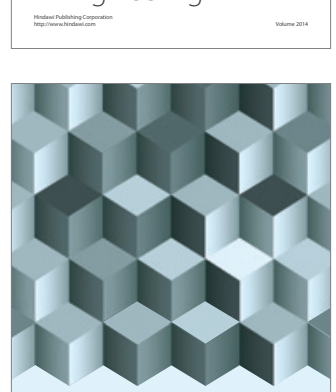

Journal of

Function Spaces
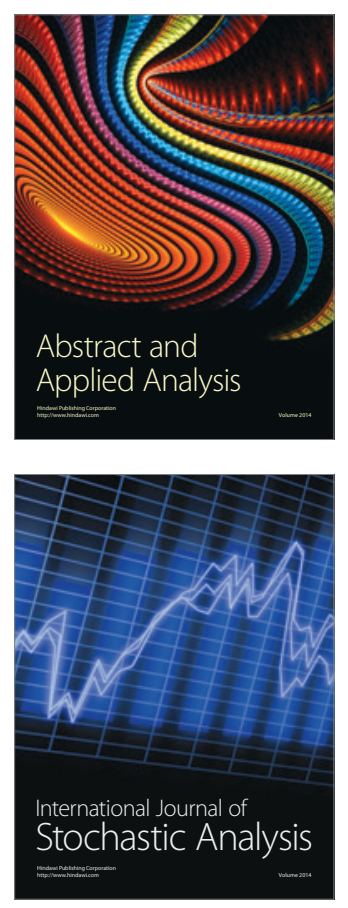

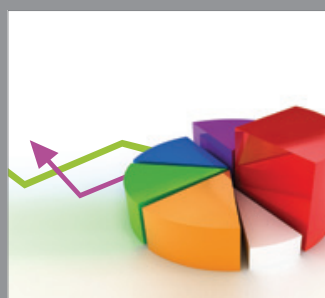

ournal of

Probability and Statistics

Promensencen
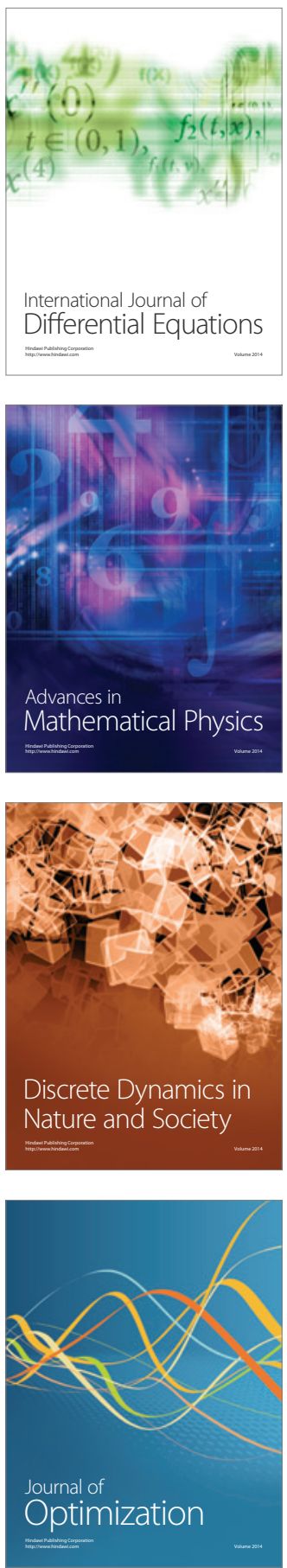